$5-8$

\title{
Cervical artificial disc replacement: still experimental? Introduction and perspectives on cervical artificial disc replacement
}

Authors Jens R Chapman, Editor-in Chief ${ }^{1}$, Daniel Riew, Guest Editor ${ }^{2}$

Institutions ${ }^{1}$ University of Washington Medicine, Seattle, Washington, WA, USA

${ }^{2}$ Washington University Orthopedics, St Louis, MO, USA

Fernstrom spherical endoprostheses, utilized in the 1960s, may have been the first artificial discs implanted in humans. These were non-articulating, ball-bearinglike devices that replaced discs and preserved motion. In 1966, Fernstrom reported the use of these devices in the cervical, as well as the lumbar spine [1] (Fig 1).

Modern cervical artificial disc replacement (C-ADR) first made its debut in 1991, with the Bristol/Cummins disc, the first of numerous articulating C-ADR devices (Fig 2). The original devices were implanted by Cummins in 20 patients, who later reported that some continued to function well, up to 12 years after implantation [2]. Results from these early articulating mechanical devices were encouraging, enough that further interest in these devices appeared very reasonable.

Over the past 20 years, the number of cervical arthroplasty devices has proliferated and now are becoming available in various shapes, materials and biomechanical concepts. Current design concepts include metal-on-metal, metal-on-plastic, non-articulating metal bonded to plastic and plastic encased in cloth. The initial cervical arthroplasty devices implanted in the United States are now a decade old. While there are a few isolated reports of osteolysis, infection and implant failure due to wear, for the most part, these devices seem to have withstood the test of time, at least in the intermediate term. Whether they will continue to fare well and have longevity comparable to the initial hip arthroplasty devices remains to be seen.

Fig 1 Fernstrom cage: this implant is widely considered to be the first disc implant and was a simple ball bearing adapted from use in the cervical and lumbar intervertebral discs.

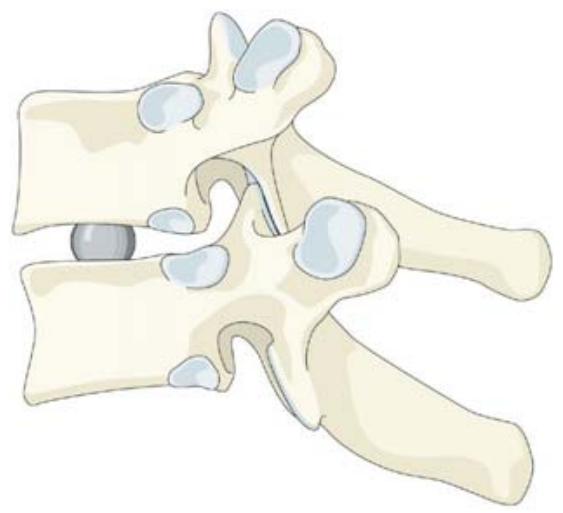

Fig 2 Cummins device: this design introduces an articulated coupling attached to the vertebral bodies with plate and screw fixation. It was modified and reintroduced as the Prestige II disc.

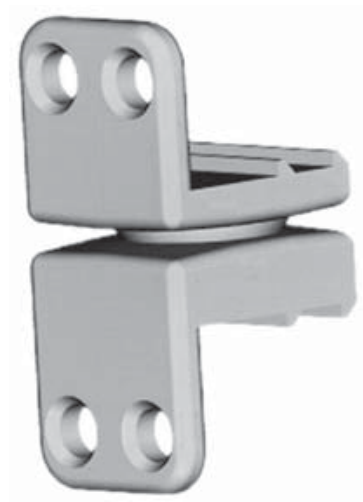


Similar to large joint arthroplasties performed on hips and knees, the differences in outcomes and revision rates for spinal arthroplasty will likely not be measurable in increments of a few years but will manifest themselves after decade-long intervals. While there are interesting similarities to the concept of hip and knee replacements, there are important differences to consider:

- Unlike hips and knees, disc arthroplasties are not synovial joints.

- Unlike hips and knees, a reasonable surgical alternative does exist-fusion surgery.

- Unlike hip and knee surgery, continued motion in an intervertebral segment may lead to a recurrence of symptoms by overgrowth of bone and fibrous tissue into adjacent neural elements.

- Unlike hip and knee replacement, cervical discs are encased in a chain of somewhat interdependent adjacent motion segments, thus increasing the focus on individual disc mechanics.

All of these considerations are important as the more longterm success of these devices will be considered. In the short term (arbitrarily defined as less than 2 years), C-ADR has certainly provided satisfactory results in all regards. As the perspective changes to longer-term follow-up factors such as reoperation rates, adjacent segment disease and patient outcomes will be increasingly scrutinized.
Short- and intermediate-term results of C-ADR certainly have been encouraging enough compared to fusion procedures to warrant greater acceptance of arthroplasty as a surgical alternative for suitably selected patients. Nonetheless, and in the face of actual published evidence, there remain significant obstacles around the world to greater utilization of C-ADR in symptomatic cervical disc disease especially from several governmental institutions and third-party payor sides. The counterarguments to disc arthroplasty are usually based upon declarations stating that these procedures are 'investigational' or 'experimental' despite a growing body of evidence to suggest that C-ADR is indeed 'safe and effective' (Figs 3-5).

\section{Indications}

The indications for cervical arthroplasty in the initial US investigational device exemption (IDE) trials were strictly limited to patients with a single-level radiculopathy or myelopathy. Patients with facet arthrosis, previous cervical surgery or multi-level disease were ineligible. There were few exemptions granted for compassionate use for patients with more than one-level disease and in some instances for patients with adjacent segment disease. While it was considered meritorious from a scientific perspective to limit the main focus of the IDE trials to the narrow indication group of single-level disc disease, this excluded a clinically relevant majority of patients, especially those with two or adjacent-to-fusion cervical spondylotic dis-
Fig 3 Prestige II disc replacement. This is a stainless steel metal-on-metal bearing articulating device with screw fixation into the vertebral body through the anterior plate.

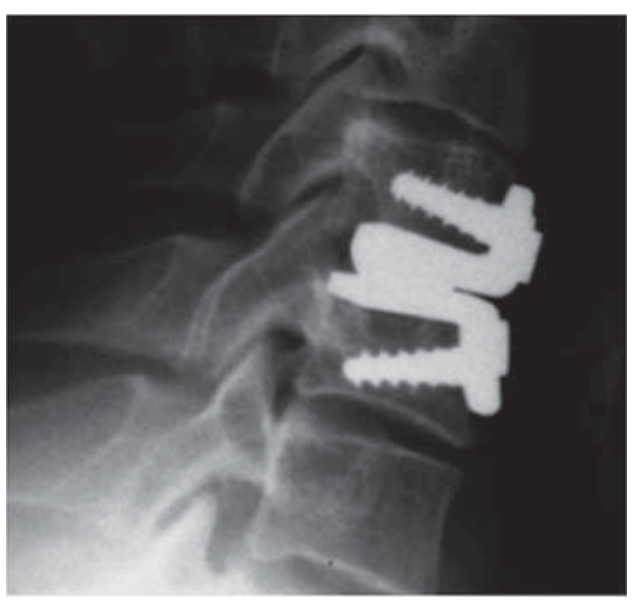

Fig 4 The Bryan disc is a composite device with articulating polyurethane ring within a saline-filled chamber. It relies on bone ingrowth along its porous-coated end plates for final fixation.

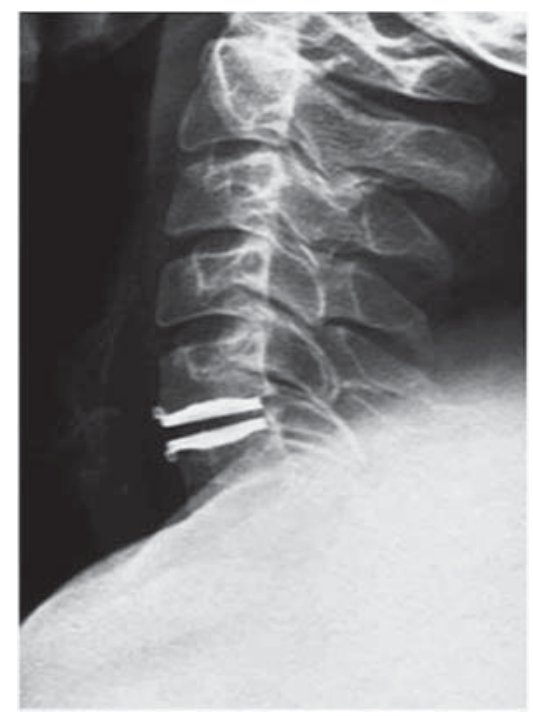

Fig 5 The Prodisc- $C$ is a chrome cobalt metal device which is secured into the host vertebrae by a keel. It features a semi-constrained design with an ultra-high density polyurethane dome articulating in an oval-shaped metal cup.

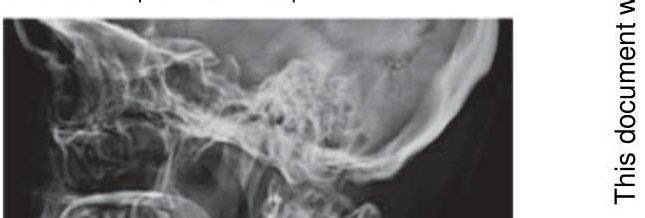


ease, from being formally studied in these pivotal first FDA-IDE trials. Thus some important opportunities to gather important insights, such as looking into multilevel disc disease progression or clinical outcomes for patients with revision surgeries using arthroplasty adjacent to fusions were unfortunately missed.

Since the Food and Drug Administration (FDA) approval of three devices, clinical indications have expanded to include patients who have had previous surgery and to multi-level disease. Mild facet arthrosis, in a patient who is asymptomatic is no longer considered an absolute contraindication. Motion-preserving disc arthroplasty should not be used in bone-forming diseases, such as ossification of the posterior longitudinal ligament (OPLL) and diffuse idiopathic skeletal hyperostosis (DISH), since bone may form around the disc. Myelopathy due to retro-discal disease can be treated effectively but not if it is due to retro-vertebral disease. Applications on patients with kyphotic neck disorders or for patients with instability of the cervical spine, as seen in trauma, infection, neoplasia etc, continue to be clear contraindications for arthroplasty.

\section{Reimbursement}

Healthcare funding has become a globally critical topic in light of serious economic uncertainty and an aging population demographic profile in many parts of the world. New health technologies face particular scrutiny because of an increasing resistance of payors to fund new technologies without immediate manifest 'evidence' of their positive impact on outcomes. In a situation like arthroplasty-where binding results may not be available for several decades, this may prove to be a detriment to patients who might actually benefit from such new technologies in the shorter and intermediate term. Reimbursement for procedures has become a powerful and readily used tool in the battle for acceptance of a new technology such as disc replacement surgery in many countries, for instance, the United States. Disc arthroplasties have indeed become an important showcase for this ongoing issue of 'how and when' should society/payors fund new healthcare technology.

"In the United States for example, initially, few, if any, nongovernmental third party payers reimbursed for cervical arthroplasty procedures. Formal publically accessible reviews of spinal arthroplasty have been conducted by governmental agencies, and when performed, have usually resulted in positive findings favoring C-ADR, such as was the case in the Washington state health technologies review in March 2009." (http://www.hta.hca.wa.gov/ documents/findings_decision_adr.pdf).

At the present time, several national insurance carriers, including United Health Care, Healthlink, Blue carriers of certain states, and governmental agencies such as Tricare/CHAMPUS (for uniformed armed services members) Medicare and Medicaid usually will reimburse for spinal arthroplasty surgery. With few exceptions, these carriers continue to follow the wording of the FDA decision and thus do not reimburse for more than a single-level disc replacement. Many of these carriers have increasingly allowed for hybrid constructs to be performed, where a one-level arthroplasty is combined with one or more levels of arthrodesis.

In addition, these carriers usually allow for the implantation of a disc adjacent to a previously fused segment, as well as in a patient who has had a previous arthroplasty, although this was not part of the original FDA trial. As the FDA-IDE trials for other devices, which were implanted at one or more levels, are getting completed and progress on to FDA approval, it may be possible to implant more than one device at a time in patients who are not Workers' Compensation or self-pay. There has been a lot of ongoing attention placed on the original three FDA IDE trials, as these are considered the most rigorous device trials of their kind to date and it appears that many other governmental agencies in other countries are somewhat influenced by the FDA decisions in the US.

\section{Future}

The topic of disc arthroplasty has invoked very diverse, sometimes outright hostile responses since its inception in Sweden over 50 years ago. Looking back, the adversity by some regarding the Fernstrom implants seems to have been more 'eminence based' preconceptions, rather than based on factual 'evidence' or documented patient safety concerns. After a lengthy period of dormancy, the last 20 years have brought about a resurgence of interest in this technology for the management of patients with unresponsive degenerative disc disease. This resurgence has gone hand-in-hand with refinements of surgical techniques and biomaterials. Anterior neck surgery, while still not perfect, has become much more predictable and safe over the last decades through more formal surgical education and many process improvements.

Just as the present day disc replacements and application techniques bear no resemblance to the original Fernstrom devices, there are important variations of technology present in the current generation of disc replacements. Important questions continue to revolve around the type of biomechanical stiffness (restrained, semi-restrained, unrestrained), its type of fixation in the host vertebrae (ingrowth, screw fixation, keel) and its wear and imaging characteristics (MRI compatibility, ceramic or polyurethane or other devices). None of the current generation arthroplasties, however, are able to mimic the natural ability of the human disc to 
absorb loads similar to a cushion-all the currently available devices will transfer axial loads to the next normal motion segment. Will this have a bearing on the rate of adjacent segment degeneration?

It stands to reason that we are just at the beginning of a further evolution of technologies where different biomaterials will be introduced; biomaterials which can absorb loads while providing secure and lasting anchoring in the host vertebrae. Biological solutions, such as the implantation of allografts and more advanced biogels are also emerging as further options in the evolution of the current generation of mechanical implants [3].

The opportunity for further device derivations is fascinating as we reflect upon the evolution of hip arthroplasty. This type of procedure is now widely recognized as one of the very most effective surgical procedures ever developed in terms of improvement of quality of life, safety and cost. During the last two decades, many modifications have been created over the initial model introduced by Sir John Charnley on the early 1960's. Non-cemented designs, changes in stem and cup architecture and many different biomaterials have been introduced to improve on the already impressive 10- and 20-year joint arthroplasty survival rates. Yet-with few exceptions-the original basic design concepts have withstood the test of time as the gold standard. Where will disc arthroplasty be in 10 and in 20 years? Will we look back at the frequently contentious fights for recognition of this generation of cervical disc arthroplasties and be glad about the advancements it has brought to the field of medicine and our patients, or will we smile about the archaic implant designs we subjected our patients to in the present day?

Or-and this is the more frightful vision-will regimented medical governance systems around the world, largely out of economic considerations, put a halt to disc replacement surgery as we know it and leave us with fusion surgery as our main tool in treating symptomatic and refractory cervical disc disease?

To this date, artificial cervical disc utilization has continued to increase steadily, as more insurance carriers reimburse for the procedure. Barring any new data that demonstrates adverse consequences in the long-term, it can be anticipated that utilization rates will continue to rise in the future. Increasing utilization rates of arthroplasty, however, will have to be mirrored by improved outcomes-reduced revision surgery rates, improved re- turn to baseline activity levels and high levels of patient safety. There is no doubt that our utilization of this new technology will be viewed under a microscope by many interested parties. Regardless of technological advances, ethical patient selection and decisionmaking, as well as technical excellence in implantation will be crucial eventual determinants of the success of cervical arthroplasties.

\section{Introduction to the EBSJ Focus Issue: "Cervical artificial disc replacement: still experimental?"}

In 2008, the precursor of EBSJ, Evidence Based Spine Surgery (EBSS) produced a focus issue with a formal systematic review of C-ADR based upon the newly published FDA-IDE studies. It concluded with a positive recommendation for disc arthroplasty over fusion based upon the then available data [4].

In this special focus issue, printed in tandem with EBSJ, we have critically reviewed the more extensive current literature on C-ADR including the more recent updates on long-term follow-up from the US FDA-IDE studies in an attempt to summarize the increasing body of literature on C-ADR. As this data has become increasingly available, we hope to provide objective and structured summaries of some of the most relevant topics surrounding disc arthroplasties to any interested party. We have been fortunate to have recruited some of the leading authorities on the topic who together with an independent Methods Core group have performed formal systematic reviews on the most pertinent current aspects of C-ADR.

We hope that your review of the findings of this group will provide additional insights into the current state of the C-ADR literature on a formal 'evidence based' format and also provide inspiration for future research.

\section{REFERENCES}

1. Fernstrom U (1966) Arthroplasty with intercorporal endoprothesis in herniated disc and in painful disc. Acta Chir Scand Suppl; 357:154-159.

2. Cummins BH, Robertson JT, Gill SS (1998) Surgical experience with an implanted artificial cervical joint. J Neurosurg; 88:943948.

3. Ruan D, He Q, Ding Y, et al (2007) Intervertebral disc transplantation in the treatment of degenerative spine disease: a preliminary study. Lancet; 369:993-999.

4. Evidence Based Spine Surgery (2008) 4:2 Special Edition. 